\title{
Power comparison among tests for fractional unit roots
}

\author{
Ignacio N. Lobato*, Carlos Velasco \\ Centro de Investigación Económica, Instituto Tecnológico Autónomo de México (ITAM), \\ Departamento de Economía, Universidad Carlos III de Madrid, Mexico
}

Received 17 October 2006; received in revised form 15 May 2007; accepted 22 June 2007

Available online 30 June 2007

\begin{abstract}
This article compares the asymptotic power properties of the Wald, the Lagrange Multiplier and the Likelihood Ratio test for fractional unit roots. The paper shows that there is an asymptotic inequality between the three tests that holds under fixed alternatives.

(C) 2007 Elsevier B.V. All rights reserved.

Keywords: Long memory; Serial correlation

JEL classification: $\mathrm{C} 22$
\end{abstract}

Several tests for the null hypothesis of unit root against the alternative of long memory have recently been proposed. Robinson (1991, 1994) proposed a Lagrange Multiplier (LM) test that was further analyzed by Tanaka (1999), whereas Lobato and Velasco (2007, LV, hereinafter) proposed an efficient Wald type test. Although both tests are locally asymptotically optimal, in the simulations in LV, it appears that the Wald test presents more empirical power than the LM test. Since LV provided no further explanation, the aim of this note is to present some analysis to help explain the apparent power superiority of the efficient Wald test over the LM test. Note that this apparent empirical power superiority also shows in the inefficient tests considered by Dolado, Gonzalo and Mayoral (2002) and Lobato and Velasco (2006).

Let $y_{t}$ denote a fractionally integrated process whose true order of integration is $d$, denoted as $I(d)$,

$\Delta^{d} y_{t} 1\{t>0\} \quad \varepsilon_{t}, \quad t \quad 1,2, \ldots$,

where $\varepsilon_{t}$ are independent and identically distributed random variables with zero mean and finite variance, and 1\{\} denotes the

\footnotetext{
* Corresponding author. Centro de Investigación Económica, Instituto Tecnológico Autónomo de México (ITAM), Av. Camino Sta. Teresa 930, México D.F. 10700, Mexico.

E-mail address: ilobato@itam.mx (I.N. Lobato).
}

indicator function. The fractional difference operator $\Delta^{d}=\left(\begin{array}{ll}1 & L\end{array}\right)^{d}$ is defined in terms of the lag operator $L$ by the formal expansion,

$$
\Delta^{\alpha}: \sum_{i}^{\infty} \pi_{i}(\alpha) L^{i},
$$

for any real $\alpha$; where for $\alpha \neq 1,2, \ldots$,

$$
\pi_{i}(\alpha) \frac{\Gamma(i \quad \alpha)}{\Gamma(i+1) \Gamma(\alpha)},
$$

and $\Gamma$ is the Gamma function, with $\Gamma(0) / \Gamma(0)=1$, so the first coefficients are $\pi_{0}(\alpha)=1$ and $\pi_{1}(\alpha)=\alpha$ : We consider testing the null hypothesis

$H_{0}: d \quad 1$,

versus either a simple alternative

$H_{A}: d \quad d_{A}<1$,

or a composite alternative

$H_{1}: d<1$.

Robinson's (1991, 1994) LM test statistic is

$\operatorname{LM}^{1 / 2} \quad T^{1 / 2}\left(\frac{\pi^{2}}{6}\right)^{-1 / 2} \sum_{j}^{T-1} j^{-1} \hat{\rho}_{\Delta y}(j)$, 
where $\hat{\rho}_{\Delta y}(j)$ denotes the sample autocorrelation of order $j$ of $\Delta y_{t}$. This test is asymptotically locally optimal in a Gaussian framework.

LV proposed to test the null hypothesis by testing the significance of the coefficient of $z_{t} 1_{1}(d)$ in the regression model

$\Delta y_{t}=\phi z_{t-1}(d)+u_{t}, \quad t=1, \ldots, T$,

where

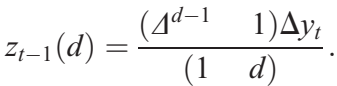

by means of a left sided test based on the $t$ ratio test statistic, denoted by $t(d)$. In practice, a consistent estimator of $d$ should be plug in.

Both tests are asymptotically equivalent in a local alternative framework, but this theoretical result does not help to un derstand the apparent power superiority of the efficient Wald test over the LM test in practice. In this note, we compare both tests in a fixed alternative framework. For completeness, we also introduce the likelihood ratio (LR) test, and establish an inequality that recalls the well known inequality among the three classical tests.

The LR test can be derived under Gaussianity assumptions from model (1), or it can be motivated by the fact that in model (3), the null and alternative hypotheses are also simple hypotheses when testing $H_{0}$ against $H_{A}$, in particular, the null corresponds to $\phi \quad 0$ and the alternative to $\phi \quad 1$. In both cases, for the simple alternative case the LR statistic is

$\mathrm{LR}=2 T \log \left(\frac{\sum\left(\Delta^{d_{A}} y_{t}\right)^{2}}{\sum\left(\Delta y_{t}\right)^{2}}\right)^{1 / 2}$.

In the composite alternative $H_{1}$ case, $d_{A}$ should be replaced by the maximum likelihood estimator of $d$.

It can be shown that, under the appropriate assumptions, the three tests share the same asymptotic null distribution, are locally asymptotically equivalent, and are consistent. Hence, in

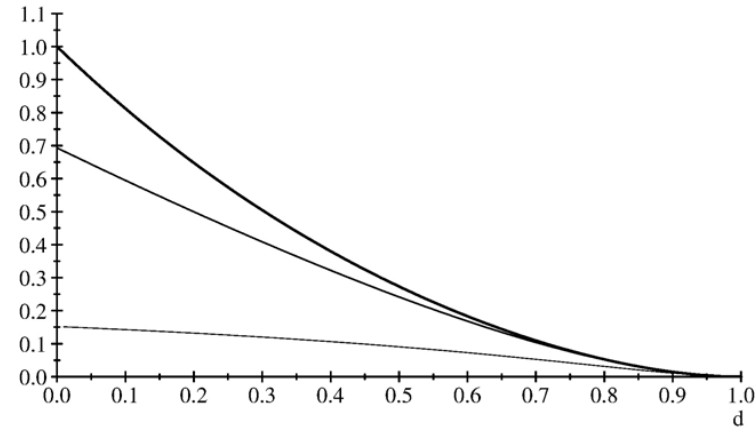

Fig. 1. Efficiency of the LM-thin line-, LR-medium line-, and $W$-thick line- tests under fixed alternatives. Respectively, these drifts are given in Eqs. (4), (5) and (6), as a function of $d$. order to compare them, we are going to use a fixed alternative framework and compute the probabilistic limit of the properly normalized test statistics. Without loss of generality, we are going to assume that $\varepsilon_{t}$ has unit variance.

First, consider the LM test statistic in Eq. (2). Since $\Delta y_{t}$ $\Delta^{1}{ }^{d} \varepsilon_{t}, d<1$; its autocorrelations are given by

$\rho_{\Delta y}(j)=\frac{\Gamma\left(\begin{array}{ll}2 & d\end{array}\right)}{\Gamma\left(\begin{array}{ll}d & 1\end{array}\right)} \frac{\Gamma\left(\begin{array}{ll}j+d & 1\end{array}\right)}{\Gamma\left(\begin{array}{ll}j+2 & d\end{array}\right)}$,

and

$$
\begin{aligned}
& \operatorname{plim}_{T \rightarrow \infty}\left(\frac{\mathrm{LM}^{1 / 2}}{T^{1 / 2}}\right)^{2}=\frac{6}{\pi^{2}}\left(\sum_{j=1}^{\infty} \frac{\rho_{\Delta y}(j)}{j}\right)^{2}
\end{aligned}
$$

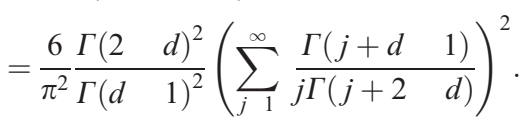

Second, using the basic relation of least squares regression theory, the efficient Wald test can be expressed as

$W=t(d)^{2}=T \frac{R^{2}(d)}{1 \quad R^{2}(d)}$,

where $R^{2}(d)$ denotes the squared sample correlation between $\Delta y_{t}$ and $z_{t}{ }_{1}(d)$. Therefore, we get that

$\operatorname{plim}_{T \rightarrow \infty} \frac{W}{T}=\frac{\varrho^{2}}{1 \varrho^{2}}$,

where $\varrho$ denotes the population correlation between reg ressand and regressor in model (3). Note that the regressand is $\Delta y_{t} \quad \Delta^{1}{ }^{d} \varepsilon_{t}$ whereas the regressor is $\left(\begin{array}{ll}1 & d\end{array}\right)^{1}\left(\begin{array}{lll}1 & \left.\Delta^{d}{ }^{1}\right) \Delta y_{t}\end{array}\right.$ $\left(\begin{array}{ll}1 & d\end{array}\right)^{1}\left(\begin{array}{lll}\Delta^{1} & d & 1\end{array}\right) \varepsilon_{t}$. It is simple to show that $\varrho^{2}$ can be written as

$\varrho^{2}=\frac{\sum_{j}^{\infty} \pi_{j}\left(\begin{array}{ll}1 & d\end{array}\right)^{2}}{1+\sum_{j=1}^{\infty} \pi_{j}\left(\begin{array}{ll}1 & d\end{array}\right)^{2}}$,

and so

$\operatorname{plim}_{T \rightarrow \infty} \frac{W}{T}=\sum_{j}^{\infty} \pi_{j}(1 \quad d)^{2}=\frac{\Gamma(3 \quad 2 d)}{\Gamma(2 \quad d)^{2}} \quad 1$.

Finally, since $T^{1} \sum\left(\Delta^{d} y_{t}\right)^{2} \rightarrow_{p} \sigma_{\varepsilon}^{2} \quad 1$, it can be shown that under $H_{A}$,

$$
\begin{aligned}
& \operatorname{plim}_{T \rightarrow \infty} \frac{\mathrm{LR}}{T}=\log \left(1+\sum_{j}^{\infty} \pi_{j}\left(\begin{array}{ll}
1 & \left.d)^{2}\right)
\end{array}\right.\right. \\
& =\log \left(\frac{\Gamma(3 \quad 2 d)}{\Gamma(2 \quad d)^{2}}\right) .
\end{aligned}
$$


A comparison of expressions (5) and (6) for the case $d=$ $1-c / \sqrt{ } T$ shows that the LR and the $W$ test are locally asymptotically equivalent, since for this case

$$
\frac{\log \left(1+\sum_{j=1}^{\infty} \pi_{j}(1-d)^{2}\right)}{\sum_{j=1}^{\infty} \pi_{j}(1-d)^{2}} \rightarrow 1,
$$

as $d \rightarrow 1$.

Fig. 1 plots the drifts of the three tests given in Eqs. (4), (5), and (6) for values of $d$ between 0 and 1. In spite of the asymptotically locally equivalence among the three tests, this Figure shows that for values of $\mathrm{d}$ distant from 1 , the $\operatorname{plim}_{T \rightarrow \infty}(\mathrm{LM} / T)$ is much lower than the $\operatorname{plim}_{T \rightarrow \infty}(W / T)$. Note that the inequalities (derived under the alternative hypothesis)

$\operatorname{plim}_{T \rightarrow \infty} \frac{W}{T} \geq \underset{T \rightarrow \infty}{\operatorname{plim}} \frac{\mathrm{LR}}{T} \geq \underset{T \rightarrow \infty}{\operatorname{plim}} \frac{\mathrm{LM}}{T}$,

can also be written as

$P(W \geq \mathrm{LR} \geq \mathrm{LM}) \rightarrow 1$ as $T \rightarrow \infty$,

which recalls the well known inequalities between the three classical tests. This analysis supports the simulations results in LV, and suggests that, when some reliable estimator of the true $d$ is available, the Wald test proposed in LV may be preferable to the optimal LM test of Robinson because of the local character of this LM test.

\section{Acknowledgments}

We thank J. Arteche, M. Avarucci, M. Delgado, J. Dolado, L. Gil-Alaña, J. Gonzalo, J. Hidalgo, L. Mayoral, P. Perron, W. Ploberger and P. Robinson for useful conversations, and seminar participants at the London School of Economics and at the 2005 Econometric Society World Congress. This research was financed by the Spanish Ministerio de Educación y Ciencia, Ref. no. SEJ2004-04583/ECON. Part of this research was carried out while Lobato was visiting Universidad Carlos III de Madrid thanks to the Spanish Secretaría de Estado de Universidades e Investigación, Ref. no. SAB2004-0034. Lobato acknowledges financial support from Asociación Mexicana de Cultura and from the Mexican Consejo Nacional de Ciencia y Tecnología (CONACYT) under project grant 41893-S.

\section{References}

Dolado, J.J., Gonzalo, J., Mayoral, L., 2002. A fractional Dickey Fuller test for unit roots. Econometrica 70, 19632006.

Lobato, I.N., Velasco, C., 2006. Optimal fractional Dickey Fuller tests. Econometrics Journal 9, 492510.

Lobato, I.N., Velasco, C., 2007. Efficient Wald tests for fractional unit roots. Econometrica 75, 575589.

Robinson, P.M., 1991. Testing for strong serial correlation and dynamic conditional heteroskedasticity in multiple regression. Journal of Econometrics 47, 6784 .

Robinson, P.M., 1994. Efficient tests of nonstationary hypotheses. Journal of the American Statistical Association 89, 14201437.

Tanaka, K., 1999. The nonstationary fractional unit root. Econometric Theory $15,249264$. 